\title{
Starlike Micelles with Starlike Interactions: A Quantitative Evaluation of Structure Factors and Phase Diagram
}

\author{
M. Laurati, J. Stellbrink, ${ }^{*}$ R. Lund, L. Willner, and D. Richter \\ Institut für Festkörperforschung, Forschungszentrum Jülich 52425 Jülich, Germany \\ E. Zaccarelli \\ Dipartimento di Fisica and INFM-CRS SOFT, Università di Roma La Sapienza, P.zza A. Moro 2, I-00185, Roma, Italy
}

(Received 30 July 2004; published 18 May 2005)

\begin{abstract}
Starlike PEP-PEO block copolymer micelles offer the possibility to investigate the phase behavior and interactions of regular star polymers (ultrasoft colloids). Micellar functionality $f$ can be smoothly varied by changing solvent composition (interfacial tension). Structure factors obtained by small-angle neutronscattering can be quantitatively described in terms of an effective potential for star polymers. The experimental phase diagram reproduces to a high level of accuracy the predicted liquid-solid transition. Whereas for intermediate $f$ a bcc phase is observed, for high $f$ the formation of a fcc phase is preempted by glass formation.
\end{abstract}

DOI: 10.1103/PhysRevLett.94.195504

Star polymers, i.e., $f$ polymer chains tethered to a central microscopic core, can be regarded as ultrasoft colloids bridging the properties of linear polymer chains and hard sphere colloids. The hybrid character is reflected in their effective potential introduced by Likos et al. [1],

$$
\begin{aligned}
\beta V(r) & =\frac{5}{18} f^{3 / 2}\left[-\ln \left(\frac{r}{\sigma}\right)+\frac{1}{1+\sqrt{f} / 2}\right], \quad r \leq \sigma \\
& =\frac{5}{18} f^{3 / 2} \frac{\sigma / r}{1+\sqrt{f} / 2} \exp \left[-\frac{\sqrt{f}(r-\sigma)}{2 \sigma}\right], \quad r \geq \sigma
\end{aligned}
$$

with $\beta=1 / k_{B} T, r$ the distance between star centers, and $\sigma$ the corona diameter. Equation (1) has given good results in modeling small-angle neutron-scattering (SANS) data of star polymer solutions [1,2]. The corresponding theoretical phase diagram [3] shows unique features, such as different crystalline phases depending sensitively on volume fraction $\phi$ and $f$. Recently the phase diagram has been revisited to include the glass transition as a result of dynamical arrest of the system [4], corroborating several experimental studies [2,5].

Unfortunately, the high interest in star polymers is not reflected in their availability. Synthesis requires considerable preparative efforts; therefore, it is of particular interest to substitute star polymers by an easy to establish model system. Ideally, this analogue should not only show the typical molecular architecture of star polymers, but also interact via the effective potential given in Eq. (1).

A few attempts to connect micellar systems to star polymers have been done, however, approaching the starlike regime [6] defined by the block ratio $N_{a} / N_{b} \gg 1$ with $a(b)$ the soluble (insoluble) block, is not trivial. McConnell et al. investigated poly(styrene)-poly(isoprene) (PS-PI) block copolymers [7]. They found experimental evidence for fcc and bcc phase formation, with crystalline geometry determined by block composition $\left(0.8 \leq N_{a} / N_{b} \leq 4\right)$. Recently PS-PI [8] and PEO-PBO [9] micelles showed a
PACS numbers: 61.25.Hq, 61.12.Ex, 64.70.Nd, 82.35.Jk

temperature induced fcc-bcc transition. This transition could be related to changes in $f$ and compared to the phase diagram of star polymers indicating some similarities in the interactions although the block ratio was rather small, $N_{a} / N_{b} \approx 2$ [8]. Micelles formed by hydrophobically modified PEO with $N_{a} / N_{b} \approx 150$ have been investigated [10], but interactions were characterized by mapping onto an equivalent hard sphere system. Therefore, a systematic study to correlate the starlike architecture of the individual micelle with starlike interactions has never been performed.

In this Letter we show that structure factors of poly(ethylene-alt-propylene)-poly(ethylene oxide) (PEPPEO) micelles (which fulfill all prerequisites of the starlike regime [11-13]) provide the basis to investigate such a correlation. SANS measurements at core contrast allow a direct determination of experimental structure factors $S(Q)$ which can be described starting from Eq. (1) with parameters $\phi, f$, and $\sigma$ given by experimental values. In addition, we can reproduce to a high level of accuracy the liquidsolid transition of the theoretical phase diagram. This was done over a wide range of $f$ and spanning the range from dilute to concentrated solutions, providing a comprehensive characterization of block copolymer micelles in terms of the microscopic, effective potential developed for star polymers.

The micellization behavior of PEP-PEO in aqueous solution is governed by the high interfacial tension, $\gamma=$ $46 \mathrm{mN} / \mathrm{m}$, between PEP and water and shows the following features [11]: (i) PEP-PEO forms micelles with the hydrophobic PEP constituting the core and the hydrophilic PEO constituting the corona. (ii) Micelles are even formed in a very asymmetric block composition with high PEO content $\left(N_{a} / N_{b} \approx 20\right)$. (iii) All micellar cores are completely segregated, i.e., not swollen by solvent. (iv) The micelles are kinetically frozen: Although chemically not linked, exchange of block copolymers between different 
micelles could not be observed [14]. This means that no free chains, i.e., no depletion effects [15] are present contrary to Ref. [8]. (v) Adjusting $\gamma$ by addition of a PEO selective cosolvent like N,N-dimethylformamide (DMF), allows the smooth varying of $f$ even in the starlike regime (where $f \sim \gamma^{6 / 5}$ was confirmed by experiments [12]).

The asymmetric PEP-PEO block copolymer under study was synthesized by anionic polymerization. To exploit $h / d$-contrast variation in SANS experiments, the individual blocks have been selectively protonated or deuterated, see Table I. Using water-DMF mixtures we prepared samples with six different $f$ going from dilute to the very concentrated regime. Since the micelles are kinetically frozen, all samples were directly prepared in the corresponding solvent mixture and annealed for at least one week, for experimental details see [12]. SANS data were corrected following standard procedures and normalized to absolute units.

The macroscopic scattering cross section, $(d \Sigma / d \Omega)(Q)$, measured by SANS can be expressed as a product of single particle contributions, the particle form factor $P(Q)$, and the structure factor $S(Q)$, which contains all information about particle interactions

$$
\left(\frac{d \Sigma}{d \Omega}\right)(Q)=N_{z} P(Q) S(Q)
$$

Here $N_{z}$ is the number density of particles. Experimental $S(Q)$ can be extracted from SANS data by dividing out the experimental $P(Q)$ measured in dilute solution. However, this procedure is only valid if particle size and shape, i.e., $P(Q)$, are unaffected by concentration. This is in general not the case for deformable particles like micelles, but we can overcome this problem by the proper application of contrast variation techniques. Performing all SANS experiments in core contrast, i.e., adjusting the scattering length

TABLE I. Characterization of PEP-PEO block copolymer and micelles. $D_{p}$ is the degree of polymerization, $x_{h}$ the protonation fraction, and $\rho$ and $\rho_{0}$ the scattering length densities of polymer blocks and solvent. $x_{\mathrm{DMF}}$ is the molar fraction of DMF in the solvent, $R_{g, \text { core }}$ and $R_{g}$ the core and overall micellar radius of gyration.

\begin{tabular}{lccccc}
\hline \hline & $M_{n}$ & $M_{W} / M_{n}{ }^{\mathrm{a}}$ & $D_{p}$ & $x_{h}$ & $\rho\left[10^{10} \mathrm{~cm}^{-2}\right]$ \\
\hline PEP (block) & 1100 & 1.06 & 15 & 1.0 & -0.31 \\
PEO (block) & 20700 & 1.04 & 436 & 0.11 & 6.32 \\
$x_{\text {DMF }}$ & $\rho_{0}\left[10^{10} \mathrm{~cm}^{-2}\right]$ & $f^{\mathrm{b}}$ & $R_{g, \text { core }}[\AA]^{\mathrm{b}}$ & $R_{g}[\AA]^{\mathrm{b}}$ & $R_{g}[\AA]^{\mathrm{c}}$ \\
0.0 & 6.33 & 136 & 31 & 194 & 192 \\
0.1 & 6.32 & 94 & 29.5 & 179 & 161 \\
0.2 & 6.34 & 82 & 29 & 164 & 154 \\
0.3 & 6.33 & 73 & 27 & 150 & 140 \\
0.4 & 6.31 & 67 & 27 & 140 & 136 \\
0.5 & 6.31 & 63 & 26 & 135 & 127 \\
\hline \hline
\end{tabular}

${ }^{\mathrm{a} O v e r a l l ~ p o l y d i s p e r s i t y ~ b y ~ S E C . ~}$

${ }^{\mathrm{b}}$ From form factor analysis.

${ }^{\mathrm{c}}$ From $\bar{\sigma}$ values obtained from fits. density $\rho_{0}$ of the solvent by use of $h / d$-isotopic mixtures to that of PEO, reduces the contrast factor between corona and solvent to zero, see Table I. Only the compact PEP core, which is completely unaffected by increasing concentration, is "visible" in the SANS experiment.

Micellar characteristics, i.e., functionality $f$, core radius $R_{c}$, and overall micellar radius $R_{m}$ have been determined as described in $[12,13] . R_{g}^{b}$ in Table I is calculated according to $R_{g}^{b}=\sqrt{5 / 11} R_{m}$ valid for star polymers, $R_{g, \text { core }}$ according to $R_{g, \text { core }}=\sqrt{3 / 5} R_{c}$ valid for solid spheres.

In this Letter we will focus on the results obtained from concentrated solutions, where particle interactions are dominant. Figure 1 shows the comparison between $S(Q)$ obtained for micelles with $f=63$ (water-DMF mixture with molar fraction $x_{\mathrm{DMF}}=0.5$ ) and a corresponding pol$\mathrm{y}$ (butadiene) (PB) star polymer in methylcyclohexane with (nominal) 64 arms [2]. Both samples have been measured at a volume fraction $\phi \approx \phi^{*}$, with $\phi^{*}=3 /\left(4 \pi R_{\underline{m}}^{3}\right) \times$ $\left(f M_{w} / \bar{d} N_{A}\right)$ being the overlap volume fraction and $\bar{d}$ the average density. All main features of the star $S(Q)$ with respect to peak positions and heights are perfectly reproduced. The only difference is found in the low $Q$ region. Here the micellar $S(Q)$ shows some increase possibly indicating the presence of large scale structures in the micellar solution. These clusters might arise either from (i) (inherent) mesoscopic heterogeneities as also found for purely repulsive star polymer solutions $[5,16]$ or (ii) from weak attractive interactions due to the decreasing solvent quality with increasing $x_{\mathrm{DMF}}$ [12]. At the moment the physical origin of clustering is not clear, but for case (i) this would only further support the analogy between PEPPEO micelles and star polymers, and for case (ii) recent theoretical results have shown that weak attractive interactions do not substantially influence the features in the region of the main structure factor peak [17]. As will be shown below on the basis of our analysis, the low- $Q$

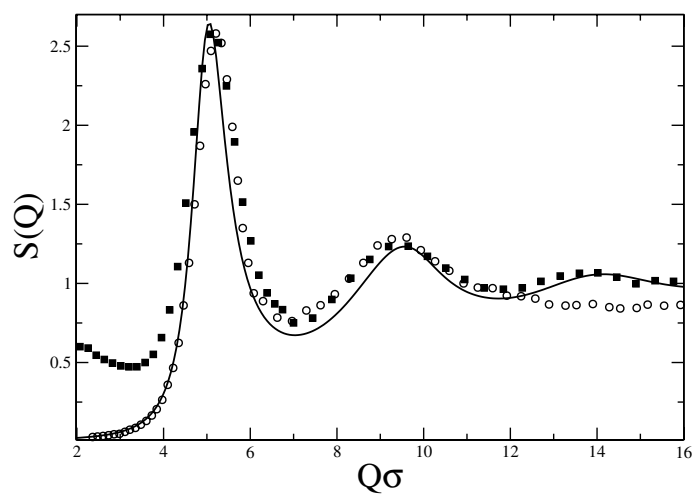

FIG. 1. $S(Q)$ for PEP-PEO micelles with $f=63$ (ם) compared to the 64 arm (nominal) PB star $(\bigcirc)$ at $\phi / \phi^{*} \approx 1$. Solid line: $S(Q)$ calculated by applying the RY closure for the $\mathrm{OZ}$ equation starting from Eq. (1), see text. 
scattering does not have major relevance on the quantitative interpretation of $S(Q)$ for $Q \geq 10^{-2} \AA^{-1}$.

Figure 1 also shows a fit to the experimental data using Eq. (1), with the corona diameter $\sigma$ being the only adjustable parameter. The theoretical $S(Q)$ was calculated by applying the self-consistent Rogers-Young (RY) closure for the Ornstein-Zernike (OZ) equation [17,18]. Different $S(Q)$ have been calculated for a fine grid of functionalities around the experimental $f=63$ to take into account the error on $f$. The experimental uncertainty on $f$ is such that no significant changes in $S(Q)$ are observed. From this fit we found a corona diameter $\sigma=226 \AA$. The radius of gyration $R_{g}$ can be calculated by $R_{g}=\sigma / 2+\sigma / \sqrt{f}$ [1]. The obtained $R_{g}=127 \pm 7 \AA$ is in very good agreement with $R_{g}=135 \pm 10 \AA$ found from independent SANS measurements of the micellar form factor [12]. Data analysis was further refined by directly fitting $(d \Sigma / d \Omega)(Q)$ including the convolution to the experimental resolution function. We assumed a compact core model for $P(Q)$, fixing the parameters to $f$ and $R_{c}$ values obtained from the characterization of the micelles. The validity of this assumption can be rationalized from Fig. 2, where the minimum arising from the solid core appears at $Q \approx 0.13 \AA^{-1}$ independent of $\phi$.

On the basis of the good agreement between theory and experiment we then applied this analysis procedure to all $f$ and $\phi$ studied. The excellent quality of the fits for $Q \geq$ $10^{-2} \AA^{-1}$ is shown in Fig. 2 for samples of different $\phi$ but same functionality $f=63$. For $\phi \leq \phi^{*}$ we found a nearly constant corona diameter giving a mean value $\bar{\sigma}=226 \pm$ $4 \AA$, which corresponds to the low concentration, unperturbed corona diameter. $\bar{\sigma}$ was then used to transform the given experimental number density $N_{z}$ to the packing fraction $\eta=N_{z} \pi / 6 \sigma^{3} . R_{g}$ values calculated from $\bar{\sigma}$ are compared to results from form factor analysis in Table I.

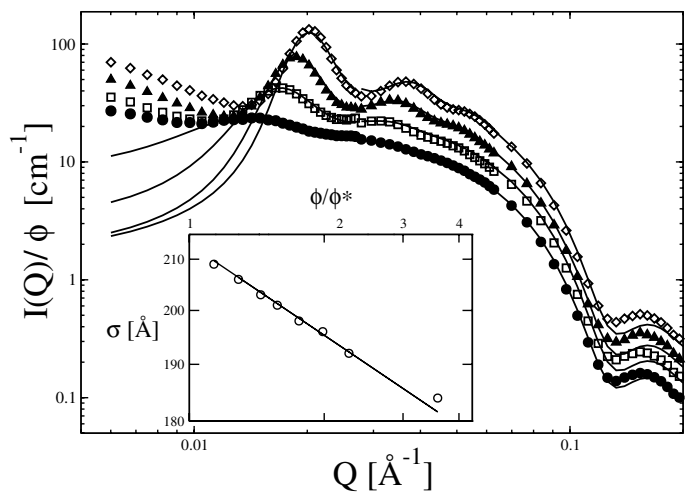

FIG. 2. Experimental intensities $I(Q)$ for $f=63$ at different $\phi$ in the fluid phase, $\phi<\phi^{*}:(\bigcirc) \phi=0.017,(\square) \phi=0.034,(\mathbf{\Delta})$ $\phi=0.051,(\diamond) \phi=0.067$ and fits (solid lines). For clarity $I(Q)$ are divided by factors: $(\diamond) 1,(\boldsymbol{\Delta}) 1.5,(\square) 2,(\boldsymbol{O}) 3$. Inset: volume fraction dependence of $\sigma$ for $f=63$, showing the expected scaling behavior $\sigma \sim \phi^{-1 / 8}$ for $\phi>\phi^{*}$.
Above $\phi^{*}$ we had to use a different approach for recalculating $\eta$. According to the Daoud-Cotton theory [19] the stars are expected to shrink as $\propto \phi^{-1 / 8}$, and indeed, the fit values for $\sigma\left(\phi \geq \phi^{*}\right)$ show exactly this scaling behavior as shown in the inset of Fig. 2. Therefore we decided to use the individual $\sigma(\phi)$ for recalculating $\eta$. Calculating $\eta$ this way for all $f$, we could then compare experimental and theoretical phase diagrams, as shown in Fig. 3. The transition from the liquid to the crystalline state (body centered cubic, bcc) predicted by theory is perfectly reproduced in experiments for $f=63$ and $f=67$. The experimental critical packing fraction for crystallization is found to be $\eta \approx 0.21$. Direct evidence for crystal formation comes from the presence of Bragg peaks in the 2-dimensional SANS pattern as shown in the inset of Fig. 3 for $f=63$ and $\phi=0.16$. Moreover crossing $\phi^{*}$ the first peak of $S(Q)$ assumes a value bigger than 2.8 , which is the minimum value for a freezing transition according to the HansenVerlet criterion [20] (see Fig. 4). The peak is further growing with increasing $\phi$. Additionally for $\phi>\phi^{*}$ a third small peak is forming, and the position ratio between the three peaks is $1: \sqrt{2}: \sqrt{3}$ corresponding to a simple cubic (sc) or bcc lattice. The A15 lattice can be directly excluded, since its additional third reflection at $\sqrt{5 / 2}$ is expected to occur in a region where the form factor is close to unity. A comparison of the lattice constants $a_{\mathrm{sc}}=270 \AA$ and $a_{\mathrm{bcc}}=381 \AA$ with $2 R_{m}=377 \AA$ at $\Phi^{*}$ excludes the sc lattice, identifying the crystal as a bcc lattice. With increasing $\eta$, no evidence for the predicted transition to a fcc phase is found in our data. However, we also note that for regular star polymers a bcc-fcc transition was never observed, probably due to the small free energy difference, $\approx k T$, between the two crystalline phases [21].

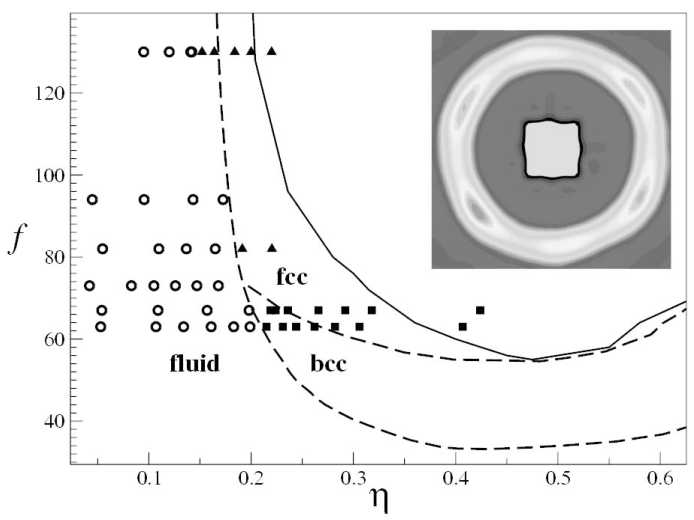

FIG. 3. Experimental phase diagram (symbols) of starlike micelles vs theoretical phase diagram (lines) of star polymers. $(\bigcirc)$ liquid, $(\boldsymbol{\square})$ bcc crystal and $(\boldsymbol{\Delta})$ gels. Dashed line represents the equilibrium phase diagram from Ref. [1]. The solid line is the RY-ideal MCT glass line from Ref. [4]. Inset: 2-dimensional SANS detector picture of $f=63, \phi=0.16$ evidencing Bragg reflections. 


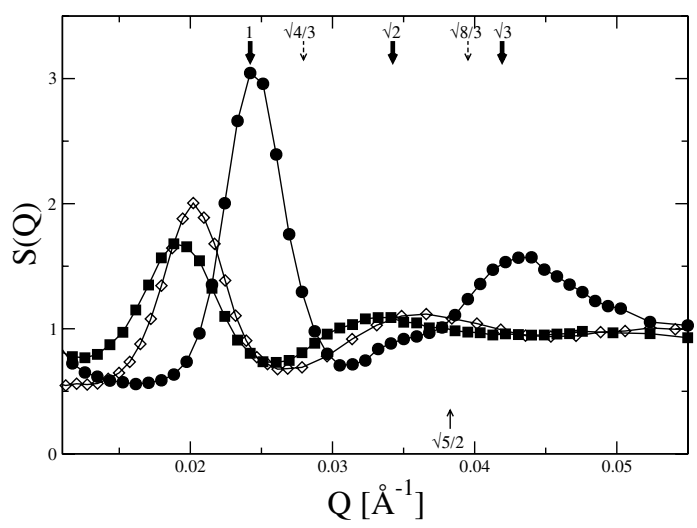

FIG. 4. Experimental $S(Q)$ for: $(\square) f=130,(\diamond) f=82$. and (O) $f=67$ at $\eta \approx 0.22$, showing the crossover from gel to crystal with decreasing $f$. $S(Q)$ for $f=67$ shows peak position ratios characteristic for bcc crystal (bold arrows). Expected peak positions for fcc (dashed arrows) and A15 crystals. Solid lines are a guide to the eye.

With increasing $f$ the experimentally observed liquidsolid transition is in nearly perfect agreement with the phase boundary predicted by theory, but instead of observing a fcc phase we observe disordered solidlike structures for all $f \geq 73$. The location of the solidus line comes from a double evidence: First from tube inversion, which means that we tested the nonflowing behavior of the sample inverting sample holders in a bath at constant temperature. In addition, preliminary rheology experiments confirm a nonzero storage modulus. Structure factor peaks for $\phi>$ $\phi^{*}$ are definitely smaller than 2.8 , excluding formation of a crystal, as shown in Fig. 4. Therefore we identify this disordered phase as a glass. The suppression of the expected equilibrium fcc phase and the early onset of glass formation at packing fractions smaller than expected from theory, might arise from an "effective" polydispersity induced by the clusters discussed above [22]. At lower $f$, where the mode coupling theory (MCT) glass line and crystal line are well separated, this effect does not play such an important role and we do observe the crystal there.

In conclusion, we have shown that starlike PEP-PEO micelles show the same interactions as star polymers, giving a significant experimental support to the picture of star polymers as ultrasoft colloids. We have been able to quantitatively describe experimental structure factors $S(Q)$ starting from a microscopic effective potential predicted for star polymers. The softness of the interaction potential between starlike micelles can be precisely varied by adjusting the interfacial tension $\gamma$ between PEP and the used solvent. In addition, we performed a detailed investigation of the phase diagram as a function of functionality $f$ and packing fractions. In particular we determined the critical packing fraction for liquid-bcc crystal transition, finding excellent agreement with theory. The simple synthesis of PEP-PEO block copolymers compared to star polymers in combination with the analogy shown in terms of effective interactions establish these micelles as an excellent alternative for investigating the phase behavior of ultrasoft colloids.

We acknowledge the allocation of SANS beam time by FZJ, ILL, and LLB and assistance during the SANS experiments by A. Radulescu (FZJ), P. Lindner (ILL), and L. Noirez (LLB). This work was supported by the Deutsche Forschungsgemeinschaft in the framework of the Transregio-SFB TR6. E. Zaccarelli acknowledges support from MIUR Cofin 2002 and FIRB. We acknowledge helpful discussions with C. N. Likos.

*Corresponding author.

Electronic address: j.stellbrink@fz-juelich.de

[1] C. N. Likos et al., Phys. Rev. Lett. 80, 4450 (1998).

[2] J. Stellbrink et al., Prog. Colloid Polym. Sci. 115, 88 (2000).

[3] M. Watzlawek, C. N. Likos, and H. Löwen, Phys. Rev. Lett. 82, 5289 (1999).

[4] G. Foffi et al., Phys. Rev. Lett. 90, 238301 (2003).

[5] M. Kapnistos et al., Phys. Rev. Lett. 85, 4072 (2000).

[6] I. W. Hamley, The Physics of Block Copolymers (Oxford University, New York, 1998).

[7] G. A. McConnell et al., Phys. Rev. Lett. 71, 2102 (1993).

[8] T. P. Lodge et al., Phys. Rev. Lett. 92, 145501 (2004).

[9] I. W. Hamley, J. Pople, and O. Diat, Colloid Polym. Sci. 276, 446 (1998).

[10] E. Beaudoin et al., Macromolecules 35, 7436 (2002).

[11] L. Willner et al., Europhys. Lett. 51, 628 (2000).

[12] R. Lund et al., Macromolecules 37, 9984 (2004).

[13] J. Stellbrink et al., J. Phys. Condens. Matter 16, S3821 (2004).

[14] R. Lund, Ph.D. thesis, Universität Münster (2004).

[15] E. Stiakakis et al., Phys. Rev. Lett. 89, 208302 (2002).

[16] J. Stellbrink, J. Allgaier, and D. Richter, Phys. Rev. E 56, R3772 (1997).

[17] F. Lo Verso, M. Tau, and L. Reatto, J. Phys. Condens. Matter 15, 1505 (2003).

[18] M. Watzlawek, H. Löwen, and C. N. Likos, J. Phys. Condens. Matter 10, 8189 (1998).

[19] M. Daoud and J.P. Cotton, J. Phys. (France) 43, 531 (1982).

[20] J. P. Hansen and L. Verlet, Phys. Rev. 184, 151 (1969).

[21] M. Watzlawek, Ph.D. thesis, University of Dusseldorf (1999).

[22] F. Sciortino et al., Phys. Rev. Lett. 93, 055701 (2004). 